\title{
The application of high resolution terahertz gas spectroscopy for medical diagnostics based on the analysis of exhaled breath and biological liquid vapor
}

\author{
Vladimir Vaks ${ }^{1}$, Mariya Chernyaeva ${ }^{1, *}$, Vladimir Anfertev ${ }^{1}$, Elena Domracheva ${ }^{1}$, \\ Oksana Garanina ${ }^{1}$, Sergey Pripolzin ${ }^{1}$, and Anton Yablokov ${ }^{1}$ \\ ${ }^{1}$ IPM RAS GSP-105, Nizhny Novgorod, 603950 Russia
}

\begin{abstract}
The results of analysing the composition of gas and liquid vapor (urine, blood) samples of human organism components by the method of high resolution terahertz spectroscopy are presented. A number of substances that are products of thermal decomposition of blood components have been detected. The proposed approach is promising for creating a method of noninvasive medical diagnostics of pathologies and diseases, including socially significant ones.
\end{abstract}

\section{Introduction}

One of the approaches with a basically new potential for the diagnostics and treatment of socially significant diseases, such as diabetes and oncologic diseases, is metabolomics. It is the field of science studying the intermediate and final metabolites in the biological system (biological cell, organ or organism). Metabolomics is capable of detecting metabolites which play an important role in the diagnostics and prognosis of a disease. Blood, urine, saliva, exhaled breath, and feces are the objects containing final and intermediate metabolic products in a human organism, which are used for detecting metabolites occurring in case of diseases and pathologies.

Hundreds of metabolites in various biological liquids of the organism were identified and quantified under the Human Metabolome Project (HMP, Canada) started in 2004. Besides, information on the previously revealed metabolites was verified and supplemented. A public electronic Human Metabolome Database (HMDB) was set up [1]. It currently contains data on more than 7900 metabolites in human organism detected in blood, urine, sweat and so on. Metabolites databases according to their identification in human excrements were created, for example, Serum Metabolome Database (SMDB) [2], which comprises data on 4229 serum/blood plasma metabolites with related concentration and possible relevance to a disease.

Methods of analysis of the component composition of exhaled breath for medical diagnostics have been under development for more than 30 years. Over 800 volatile compounds [3], products of physiological and biochemical processes in the organism that

* Corresponding author: masha@ipmras.ru 
may be present in human breath are known today. Some metabolites indicate specific pathological states. Complex studying the content of the metabolites of pathological states in patients' breath and of its biological liquids (urine, saliva, etc.) is a promising direction of research [4].

\section{Objects of research and experimental technique}

The objects of the study were human exhaled breath and biological liquids (urine, blood). Samples were taken from conditionally healthy volunteers as well as from people with a diagnosed disease (diabetes).

Multicomponent gas mixtures of biological origin were analysed by the method of nonstationary subterahertz and terahertz spectroscopy [5] based on the effect of freedamping polarization. This effect is, actually, the following: if polarization is produced in a gas sample under resonance conditions, when the generator is out of resonance, the polarization wave will emit the same field mode that has excited it. This is done by periodic switching of the phase or frequency of probe radiation resonantly interacting with the medium, which results in nonstationary radiation and absorption and, hence, in periodic generation and decay of induced macroscopic polarization. Spectrometers provide sensitivity close to the theoretical maximum with a resolution limited only by the Doppler effect and can record fast processes. The sensitivity is preserved even with a substantial decrease in gas pressure (down to hundredth and thousandth of Torr) and is approximately $0.2 \mathrm{ppb}$ in the scanning mode for some gases. The instruments can be implemented in two forms: a phase manipulation spectrometer and a fast frequency sweep spectrometer [6]. The phase manipulation mode is good for measurements at a specific frequency and is needed for monitoring an increase or a decrease in the concentration of the chosen substance. The fast frequency sweep mode allows several absorption lines to be measured simultaneously in real time and several substances to be detected all at once.

\section{Results of analysing component composition of gas and liquid vapor samples of human components}

The level of acetone at frequencies of $150.537 \mathrm{GHz}$ and $151.647 \mathrm{GHz}$ in samples of exhaled breath and urine taken from the same type 2 diabetes patients was measured in collaboration with Almazov National Medical Research Centre. Simultaneous analysis of the samples of exhaled breath and urine of diabetes patients revealed that the acetone level in the urine was much higher than in the exhaled breath, in some cases an order of magnitude higher.

Capillary blood vapor of conditionally healthy volunteers was analysed using the following procedure.

Immediately after blood sampling its spectrum was measured. Capillary blood was poured into a test tube, vacuum pumped, vacuum dried to exclude the influence of water vapor, and heated to decompose the dry residue. The measurements were made with the subterahertz fast frequency sweep spectrometer. A data file usually includes recordings of a selected or of the total operating range of the spectrometer. Therefore, substances can be identified by absorption lines appearing in the total spectrum of the sample and the dynamics of concentrations in a mixture can be traced by comparing several data files.

The substances detected as a result of decomposition of capillary blood samples of three conditionally healthy volunteers are presented in Table 1 . The substances were identified using spectral databases $[7,8]$. 
Table 1. Substances detected as a result of thermal decomposition of capillary blood samples of 3 conditionally healthy volunteers.

\begin{tabular}{|c|c|c|c|}
\hline \multirow{2}{*}{ Substance } & \multicolumn{3}{|c|}{ Number of lines recorded in the spectrum } \\
& Sample 1 (male) & Sample 2 (female) & Sample 3 (female) \\
\cline { 2 - 4 } & 1 & 1 & 1 \\
\hline $\mathrm{H}_{2} \mathrm{~S}$ & 1 & 1 & 1 \\
\hline $\mathrm{H}_{2} \mathrm{~S}-34$ & 2 & 3 & 3 \\
\hline $\mathrm{OCS}$ & 2 & 1 & 1 \\
\hline $\mathrm{C}_{4} \mathrm{H}_{9} \mathrm{CN}$ & 10 & 55 & 18 \\
\hline $\mathrm{CH}_{3} \mathrm{CHO}$ & 4 & 12 & 11 \\
\hline $\mathrm{HNCO}$ & 1 & - & - \\
\hline $\mathrm{HCOOH}$ & 1 & 1 & data \\
\hline $\mathrm{NH}_{3}$ & 2 & - & 1 \\
\hline $\mathrm{CH}_{3} \mathrm{OCHO}$ & - & 8 & - \\
\hline $\mathrm{CH}_{3} \mathrm{CN}$ & 1 & 4 & 1 \\
\hline $\mathrm{C}_{3} \mathrm{H}_{7} \mathrm{CN}$ & - & 3 & 4 \\
\hline $\mathrm{C}_{2} \mathrm{H}_{3} \mathrm{CN}$ & - & - & - \\
\hline $\mathrm{CH}_{3} \mathrm{SH}$ & & & \\
\hline
\end{tabular}

The first substance appearing during sample heating is hydrogen sulfide $\left(\mathrm{H}_{2} \mathrm{~S}\right)$, including its isotopologue $\mathrm{H}_{2} \mathrm{~S}-34$. Then there appear OCS and nitrile lines. In our experiment, series of absorption lines of isocyanic acid (HNCO) and acetaldehyde $(\mathrm{CH} 3 \mathrm{CHO})$ were identified in the spectra of all samples.

For some substances, for example, $\mathrm{H}_{2} \mathrm{~S}, \mathrm{H}_{2} \mathrm{~S}-34$, and $\mathrm{NH}_{3}$, only one line was recorded, as there are no more high intensity lines of this substance in the operating range of the spectrometer.

For other substances, for instance, for formic acid the absorption lines were present in one sample only.

The composition of capillary blood sample 1 was compared with the blood plasma sample of the same volunteer. During blood plasma heating, the following compounds were detected: hydrogen sulfide $\left(\mathrm{H}_{2} \mathrm{~S}, \mathrm{H}_{2} \mathrm{~S}-34\right)$, nitriles $\left(\mathrm{C}_{3} \mathrm{H}_{7} \mathrm{CN}, \mathrm{C}_{2} \mathrm{H}_{3} \mathrm{CN}, \mathrm{C}_{4} \mathrm{H}_{9} \mathrm{CN}\right)$, aldehydes $\left(\mathrm{C}_{2} \mathrm{H}_{3} \mathrm{CHO}, \mathrm{CH}_{3} \mathrm{CHO}\right), \mathrm{OCS}, \mathrm{CH}_{3} \mathrm{SH}, \mathrm{HNCO}$, and $\mathrm{NH}_{3}$. The main feature of the revealed plasma composition as distinct from the capillary blood is the presence of methyl mercaptan $\left(\mathrm{CH}_{3} \mathrm{SH}\right)$. Examples of recording parts of the spectra with lines of acetonitrile and isocyanic acid are shown in figs. 1, 2 .

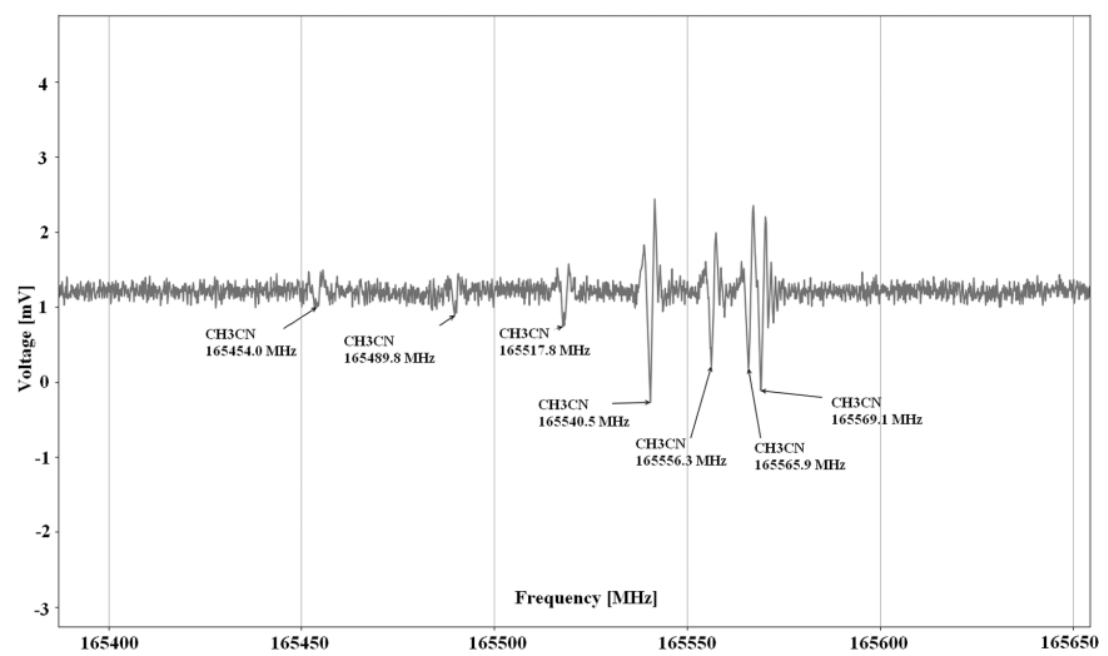

Fig. 1. Recording of the part of the spectrum with acetonitrile $\left(\mathrm{CH}_{3} \mathrm{CN}\right)$ lines at frequencies $165.454 \mathrm{GHz}, 165.489 \mathrm{GHz}, 165.518 \mathrm{GHz}, 165.540 \mathrm{GHz}, 165.556 \mathrm{GHz}, 165.566 \mathrm{GHz}$, and $165.569 \mathrm{GHz}[7,8]$. 


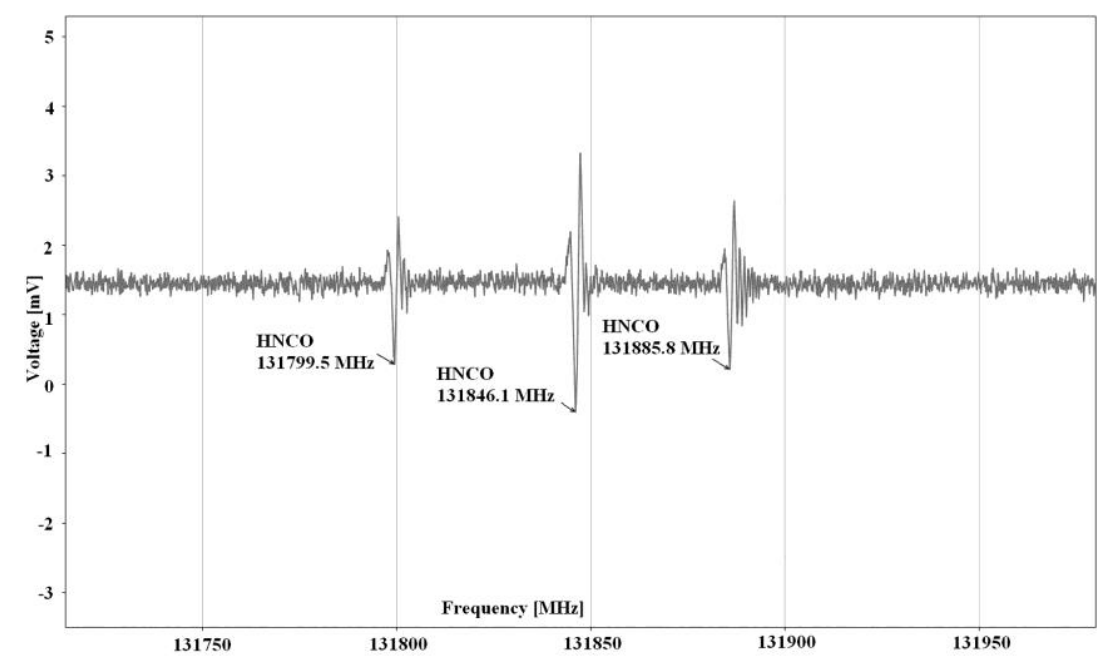

Fig. 2. Recording of the part of the spectrum with lines of isocyanic acid $(\mathrm{HNCO})$ at frequencies $131.800 \mathrm{GHz}, 131.846 \mathrm{GHz}$, and $131.886 \mathrm{GHz}[7,8]$.

Not all substances can be recorded simultaneously. Some substances start to decompose and their decay products become volatile at lower temperatures than others. Some substances can be detected almost throughout the heating period (several hours), but their amount gradually increases $\left(\mathrm{CH}_{3} \mathrm{CN}\right)$ or decreases $\left(\mathrm{H}_{2} \mathrm{~S}\right)$. The dynamics of hydrogen sulfide during plasma sample heating is shown in fig. 3 .

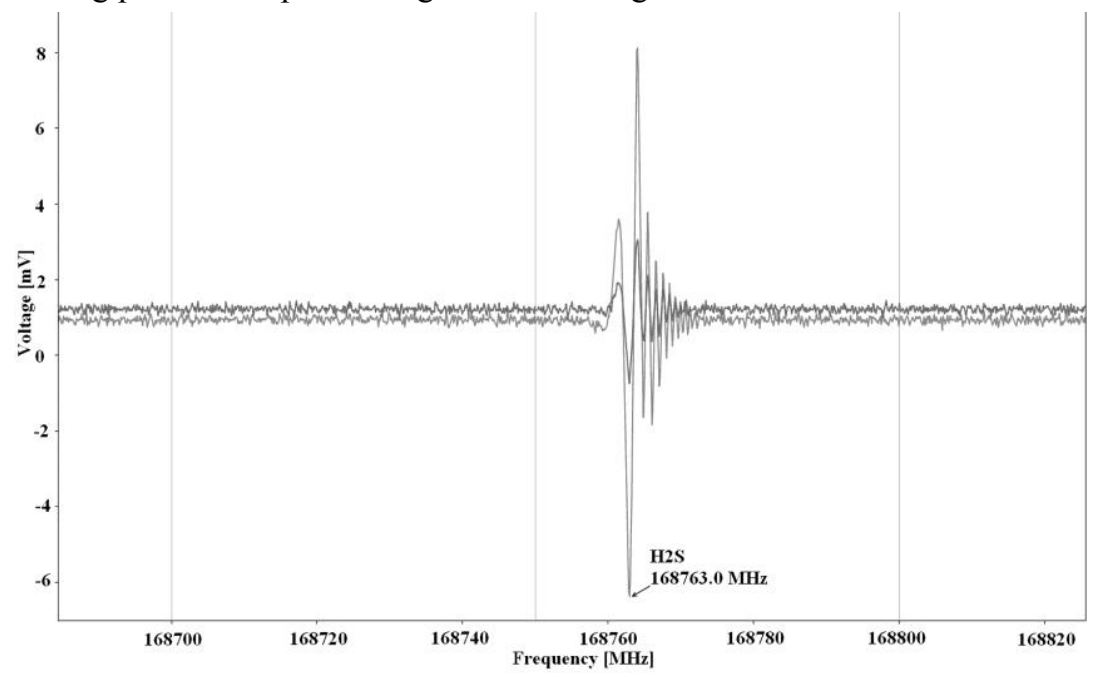

Fig. 3. Recording of hydrogen sulfide absorption line demonstrating dynamics of its concentration during plasma sample heating at a frequency of $168.763 \mathrm{GHz}[7,8]$ (grey - beginning of heating, black - heating for a long time).

The presence of isocyanic acid and ammonia in the composition of capillary blood and blood plasma samples may be attributed to thermal decomposition of carbamide at heating above its boiling temperature (with decomposition) that is $174{ }^{\circ} \mathrm{C}[9]$ :

$$
\mathrm{CO}(\mathrm{NH} 2)_{2} \rightarrow \mathrm{NH}_{3}+\mathrm{HNCO}
$$

The presence of sulfur compounds such as hydrogen sulfide and methyl mercaptan may be specified by thermal decomposition of sulfur amino acids (methionine, cysteine, cystine) in the blood protein composition. Methionine is an essential amino acid that is not 
synthesized in human organism. It is obtained from the food and is a source of sulfur for cysteine biosynthesis. When sulfur amino acids were heated up to temperatures of about $240^{\circ} \mathrm{C}$, hydrogen sulfide was registered in their mass spectrum, primarily in cystine and cysteine, and in a smaller quantity in methionine. Besides, at thermal decomposition methionine is a source of methyl mercaptan and ammonia, and cysteine may be a source of acetonitrile $\left(\mathrm{CH}_{3} \mathrm{CN}\right)$ [10]. The presence of methyl mercaptan in a sample of blood plasma and absence in capillary blood may be explained by increased concentration of proteins and, hence, their amino acid constituents in the blood plasma.

The reasons for some substances to emerge in a mixture during thermal decomposition of blood deserve special consideration. Investigation of the blood of conditionally healthy patients as well as patients with diabetes and cancer will be continued aimed at detecting specific proteins typical for particular diseases that may be recorded by products of their thermal decomposition.

\section{Conclusion}

We have reported the results of analysing the composition of exhaled breath, blood and urine vapor using the method of nonstationary spectroscopy. The nonstationary subterahertz and terahertz spectroscopy with a sensitivity of about $0.2 \mathrm{ppb}$ (for some gases), high selectivity, ability to estimate the concentration of the studied substances, and easy use make it a unique diagnostic method for investigating multicomponent gas mixtures, such as exhaled breath or vapor of biological liquids.

The research was performed under the State assignment 0035-2014-0206 and with financial support from the RFBR (grant No. 18-42-520050 p_a, No. 17-00-00184 KOMFI, No 18-52-16017), by the state program of IPM RAS (2019) (0030-2019-0021-C-01).

\section{References}

1. D.S. Wishart, Y.D. Feunang, A. Marcu, A.C. Guo, K. Liang et al., Nucleic Acids Res. 46(D1), D608-617 https://doi.org/10.1093/nar/gkx1089 (2018)

2. N. Psychogios, D. D. Hau, J. Peng, A. C. Guo, R. Mandal et al., PLoS One. 6(2), e16957 https://doi.org/10.1371/journal.pone.0016957 (2011)

3. E.V. Stepanov, Diode Laser Spectroscopy and Analysis of Molecules-Metabolomes, Fizmatlit, Moscow, [in Russian] (2009)

4. A. Amann, B. L. Costello, W. Miekisch, J. Schubert, B. Buszewski et al., J. Breath Res.. 8, 034001 http://dx.doi.org/10.1088/1752-7155/8/3/034001 (2014)

5. V. Vaks, J. Infrared Millim. Terahertz Waves. 33 (1), 43-53 https://doi.org/10.1007/ s10762-011-9846-x (2012)

6. V. L. Vaks, V. A. Anfertev, V. Yu. Balakirev, S. A. Basov, E. G. Domracheva et al., Uspekhi Fiz. Nauk, , DOI: 10.3367/UFNr.2019.07.038613 [in Russian] (to be published)

7. H. M. Pickett, E. A. Cohen, B. J. Drouin, J. C. Pearson et al., Submillimeter, millimeter, and microwave spectral line catalog. JPL Molecular Spectroscopy, California Institute of Technology. http://spec.jpl.nasa.gov/ftp/pub/catalog/catform.html (2003)

8. C. P. Endres, S. Schlemmer, P. Schilke, J. Stutzki, H. S. P. Müller, J. Mol. Spectrosc. 327, 95-104 https://doi.org/10.1016/j.jms.2016.03.005 (2016)

9. N. F. Timerbayev, R. G. Safin, A. R. Sadrtdinov, Vestnik KTU. 11, 243-246 [in Russian] (2010)

10. V.A. Yablokov, Ya.A. Vasina, I.A. Zelyaev, and S.V. Mitrofanova. Russian Journal of General Chemistry. 79(6), 1141-1145 [in Russian] (2009) 